\title{
Confinement, Chiral Symmetry Breaking and it's Restoration us- ing Dual QCD Formalism
}

\author{
Garima Punetha ${ }^{a}$ and H. C. Chandola \\ Center of Advanced Study, Department of Physics, Kumaun University Nainital-263001, India
}

\begin{abstract}
Utilizing the dual QCD model in term of magnetic symmetry structure of nonAbelian gauge theories, the dynamical chiral-symmetry breaking using Schwinger-Dyson equation has been investigated. A close relation among the color confinement and chiralsymmetry breaking has been observed and demonstrated by computing dynamical parameters. The recovery of the chiral symmetry has also been discussed at finite temperature through the variation of quark mass function and quark condensate which gradually decreases with temperature and vanishes suddenly near the critical temperature.
\end{abstract}

\section{Introduction}

Color confinement and chiral-symmetry breaking are two outstanding non-perturbative phenomena in quantum chromodynamics (QCD) and their relation have been studied as one of the important difficult problems in theoretical particle physics. In this direction, the dual superconductor picture for the Yang-Mills theory [1-5] is an attractive hypothesis for explaining quark confinement and for this mechanism to work, the existence of chromomagnetic monopole and their condensation play a leading role to cause the dual Meissner effect. In QCD, chiral symmetry is approximately realized at the Lagrangian level and is broken by the strong interaction at zero temperature. On the other hand, at finite temperature, the broken symmetries are restored. As a theoretical approach to understand the phase structure of QCD, the present paper, therefore, deals to present a gauge independent field decomposition formalism in which the QCD potential splits into the non-topological abelian part and the topological monopole part to establish the color confinement and chiral symmetry breaking using Schwinger-Dyson equation (SDE) method.

\section{Dual QCD Formulation and Confinement}

For the case of the simplest non-abelian gauge group, $G=S U(2)$ [6-12] with its little group $H$ as $U(1)$, the multiplet $\hat{m}$ may thus be viewed to identify the homotopy of the mapping $\pi_{2}\left(S^{2}\right)$ in the following form, $\hat{m}: S_{R}^{2} \rightarrow S^{2}=S U(2) / U(1)$, where, $S_{R}^{2}$ is the two dimensional sphere of the three dimensional space and $S^{2}$ is the group coset space completely fixed by $\hat{m}$. The typical gauge potential and field strength may then identified as

$$
\mathbf{W}_{\mu}=A_{\mu} \hat{m}-g^{-1}\left(\hat{m} \times \partial_{\mu} \hat{m}\right) \text { and } \mathbf{G}_{\mu \nu}=\partial_{\mu} \mathbf{W}_{\nu}-\partial_{\nu} \mathbf{W}_{\mu}+g\left(\mathbf{W}_{\mu} \times \mathbf{W}_{\nu}\right)=\left(F_{\mu \nu}+B_{\mu \nu}^{(d)}\right) \hat{m}
$$

\footnotetext{
ae-mail: garimapunetha@gmail.com
} 
where, $A_{\mu} \equiv \hat{m} \cdot \mathbf{W}_{\mu}$ is the Abelian component of $\mathbf{W}_{\mu}$, while the second part on the right hand side is completely determined by the magnetic symmetry and is of topological origin. The topological structure of the theory may be then brought into dynamics explicitly by performing the separation of gauge fields in the magnetic gauge obtained by rotating $\hat{m}$ to the third direction in isospace such that, $\hat{m} \stackrel{U}{\longrightarrow} \hat{\xi}_{3}=U \hat{m}=\left(\begin{array}{lll}0 & 0 & 1\end{array}\right)^{T}$ along with the parameterisation $\hat{m}=(\sin \alpha \cos \beta, \sin \alpha \sin \beta, \cos \alpha)^{T}$. The parameterisation with the choice $U=\exp \left(-\alpha t_{2}-\beta t_{3}\right)$ then leads to the expression of the potential and the corresponding field strength as

$$
W_{\mu} \stackrel{U}{\longrightarrow} W_{\mu}^{\prime}=\left(A_{\mu}+B_{\mu}\right) \hat{\xi}_{3} \text { and } G_{\mu \nu} \stackrel{U}{\longrightarrow} G_{\mu \nu}=\left(F_{\mu \nu}+B_{\mu \nu}^{(d)}\right) \hat{\xi}_{3},
$$

where, the part $B_{\mu \nu}^{(d)}$ may now be identified as $B_{\mu \nu}^{(d)}=-g^{-1} \sin \alpha\left(\partial_{\mu} \alpha \partial_{\nu} \beta-\partial_{\nu} \alpha \partial_{\mu} \beta\right)=\partial_{\mu} B_{v}-\partial_{v} B_{\mu}$. In order to explore the typical dual QCD vacuum properties and the dynamics of the system, the Lagrangian in the quenched approximation may be expressed as [6]

$$
\mathcal{L}_{d}^{(m)}=-\frac{1}{4} B_{\mu \nu}^{2}+\left|\left[\partial_{\mu}+i \frac{4 \pi}{g} B_{\mu}^{(d)}\right] \phi\right|^{2}-V\left(\phi^{*} \phi\right) .
$$

Using $V\left(\phi^{*} \phi\right)=3 \lambda \alpha_{s}^{-2}\left(\phi^{*} \phi-\phi_{0}^{2}\right)^{2}$ potential the Lagrangian given by equation (3) generate the dynamical symmetry breaking and leads to the following field equations given as below [10]

$$
\begin{gathered}
\left(\partial_{\mu}-i \frac{4 \pi}{g} B_{\mu}^{(d)}\right)\left(\partial_{\mu}+i \frac{4 \pi}{g} B_{\mu}^{(d)}\right) \phi+96 \lambda \alpha_{s}^{-2}\left(\phi^{*} \phi-\phi_{0}^{2}\right) \phi=0, \\
\partial^{v} B_{\mu \nu}+\frac{i 4 \pi}{g}\left(\phi \partial_{\mu} \phi^{*}-\phi^{*} \partial_{\mu} \phi\right)-\frac{32 \pi^{2}}{g^{2}} B_{\mu}^{(d)} \phi \phi^{*}=0 .
\end{gathered}
$$

Utilizing the asymptotic solutions of the associated dual QCD fields, the energy per unit length of the resulting flux tube configuration may be derived as [10]

$$
k=2 \pi \int_{0}^{\infty} \rho d \rho\left[\frac{n^{2} g^{2}}{32 \pi^{2} \rho^{2}}\left(F_{\lambda}^{\prime}(\rho)\right)^{2}+\frac{n^{2} g^{2}}{16 \pi^{2} \rho^{2}} F_{\lambda}^{2}(\rho) \chi^{2}(\rho)+\left(\chi^{\prime}(\rho)\right)^{2}+\frac{48 \lambda \pi^{2}}{g^{4}}\left(\phi^{*} \phi-\phi_{0}^{2}\right)^{2}\right] .
$$

In terms of a set of more convenient parameters, $r=2 \sqrt{3 \lambda} \alpha_{s}^{-1} \phi_{0} \rho, F_{\lambda}(r)=\left(4 \pi \alpha_{s}^{-1}\right)^{\frac{1}{2}} \rho B(\rho), H_{\lambda}(r)=$ $\phi_{0}^{-1} \chi(\rho)$, eq. (5) may be reduced to a simple form as [10]

$$
k=\gamma \phi_{0}^{2}, \text { with, } \gamma_{\lambda}=2 \pi \int_{0}^{\infty} r d r\left[\frac{6 \lambda}{g^{2}} \frac{\left(F_{\lambda}^{\prime}\right)^{2}}{r^{2}}+\frac{\left(n+F_{\lambda}\right)^{2}}{r^{2}} H_{\lambda}^{2}+\left(H_{\lambda}^{\prime}\right)^{2}+\frac{\left(H_{\lambda}^{2}-1\right)^{2}}{4}\right] .
$$

Using the numerical computation of equation (6) for $\gamma_{\lambda}$, we obtain the characteristics mass scales, the scalar $\left(m_{\phi}\right)$ and vector $\left(m_{B}\right)$ glueball masses for some typical values of coupling in infrared sector of QCD and are summarized in Table $1[7,10]$.

\section{Chiral Symmetry Breaking using Dual QCD Formalism}

In the low energy sector the dual gluon propagator leads to a linear confinement potential and the chiral symmetry associated with the quarks is broken dynamically. The SDE for quark propagator is expressed as

$$
S^{-1}(p)=Z_{2}\left(i \gamma \cdot p+m_{b}\right)+Z_{1} \int \frac{d^{4} q}{(2 \pi)^{4}} g^{2} \gamma^{\mu} \frac{\lambda^{a}}{2} S(q) \Gamma^{v b} \times D_{\mu \nu}^{a b}(p-q),
$$


Table 1. The masses of vector and scalar glueball as functions of $\alpha_{s}$ using Quartic potential.

\begin{tabular}{llllllll}
\hline$\alpha_{s}$ & $\gamma$ & $\phi_{0}$ & $m_{B}(\mathrm{GeV})$ & $m_{\phi}(\mathrm{GeV})$ & $\lambda_{Q C D}^{(d)}\left(\mathrm{GeV}^{-1}\right)$ & $\xi_{Q C D}^{(d)}\left(\mathrm{GeV}^{-1}\right)$ & $\kappa_{Q C D}^{(d)}$ \\
\hline 0.12 & 8.30 & 0.143 & 2.11 & 4.20 & 0.473 & 0.238 & 2 \\
\hline 0.22 & 7.89 & 0.149 & 1.51 & 2.2 & 0.662 & 0.450 & 1.5 \\
\hline 0.47 & 6.28 & 0.167 & 1.21 & 1.22 & 0.826 & 0.819 & 1 \\
\hline 0.96 & 5.40 & 0.181 & 0.929 & 0.655 & 1.07 & 1.53 & 0.7 \\
\hline
\end{tabular}

where $S(p)$ is the full quark propagator, $D_{\mu \nu}^{a b}$ is the dressed gluon propagator, $\Gamma^{\nu b}$ is the dressed quarkgluon vertex, $m_{b}$ is the bare mass of the quark, $Z_{1}$ is the quark-gluon vertex renormalization and $Z_{2}$ is the quark wave function renormalization. In solving SDE, a system of two coupled integral equation for $A\left(p^{2}\right)$ and $M\left(p^{2}\right)$ are obtained and expressed as

$$
\begin{gathered}
{\left[A\left(p^{2}\right)-1\right] p^{2}=\frac{4}{3} g^{2} \beta \int \frac{d^{4} q}{(2 \pi)^{4}} \frac{A\left(q^{2}\right)}{q^{2} A^{2}\left(q^{2}\right)+B^{2}\left(q^{2}\right)}\left[p q+\frac{q(p-q)(p-q) p}{(p-q)^{2}}\right],} \\
M\left(p^{2}\right)=4 g^{2} \beta \int \frac{d^{4} q}{(2 p i)^{4}} \delta^{4}(p-q) \frac{B\left(q^{2}\right)}{q^{2} A^{2}\left(q^{2}\right)+B^{2}\left(q^{2}\right)},
\end{gathered}
$$

where the gluon propagator $[13,14]$ is given as $D_{\mu \nu}^{a b}(q)=\delta^{a b}\left(\delta_{\mu \nu}-\frac{q_{\mu} q_{v}}{q^{2}}\right) \beta \delta^{4}(q)$. The equation for $M\left(p^{2}\right)$ gives the momentum dependent quark mass function generated dynamically and indicates the breaking of the chiral symmetry in the dual QCD vacuum. The solution of the above coupled integral equations determined in [15-18] showing the validity in the infrared region of QCD expressed as

$$
M\left(p^{2}\right)=\sqrt{\frac{2 m_{B}^{2} \alpha_{s}}{\pi}-\frac{4 p^{2}}{9}} \quad p^{2}<\frac{9}{4}\left(\frac{2 m_{B}^{2} \alpha_{s}}{\pi}\right) \text { and } M\left(p^{2}\right)=0 \quad p^{2}>\frac{9}{4}\left(\frac{2 m_{B}^{2} \alpha_{s}}{\pi}\right)
$$

The non-trivial solution of the SDE is denoted by the solid curve in Figure 1 through the variation of $M\left(p^{2}\right)$ with momentum scale $(p)$ for different values of $\alpha_{s}$. The above equation reduces to the square of dynamical quark mass as $M^{2}(0)=\frac{2 m_{B}^{2} \alpha_{s}}{\pi}$ which consists of two parameters $m_{B}$ and $\alpha_{s}$ in the infrared region. The relationship between quark condensate $(<q \bar{q}>)$ and $M\left(p^{2}\right)$ for dual QCD is expressed as [19]

$$
<q \bar{q}>=-\frac{2}{4 \pi^{2}} \int d p^{2} \frac{p^{2} M\left(p^{2}\right)}{\frac{4}{9} p^{2}+M^{2}\left(p^{2}\right)}=-\frac{27}{40 \pi^{2}}\left(\frac{2 m_{B}^{2} \alpha_{s}}{\pi}\right)^{3 / 2} .
$$

The corresponding plot of dynamical quark mass $(M(0))$ and $<q \bar{q}>$ as a function of $\alpha_{s}$ have been shown is Figure 1 and their numerical values are estimated in Table 2 for various coupling. The

Table 2. The dynamical quark mass $M(0)$ and quark condensate $<q \bar{q}>$ as functions of $\alpha_{s}$.

\begin{tabular}{llll}
\hline$\alpha_{s}$ & $m_{B}(\mathrm{GeV})$ & $M(0)(\mathrm{GeV})$ & $<q \bar{q}>\left(\mathrm{GeV}^{3}\right)$ \\
\hline 0.12 & 2.11 & 0.583 & 0.0135 \\
\hline 0.22 & 1.51 & 0.565 & 0.0123 \\
\hline 0.47 & 1.21 & 0.662 & 0.0198 \\
\hline 0.96 & 0.929 & 0.726 & 0.0262 \\
\hline
\end{tabular}

non-zero value of $\left\langle q \bar{q}>\right.$ in condensed QCD vacuum appears strong for the large value of $m_{B}$, 

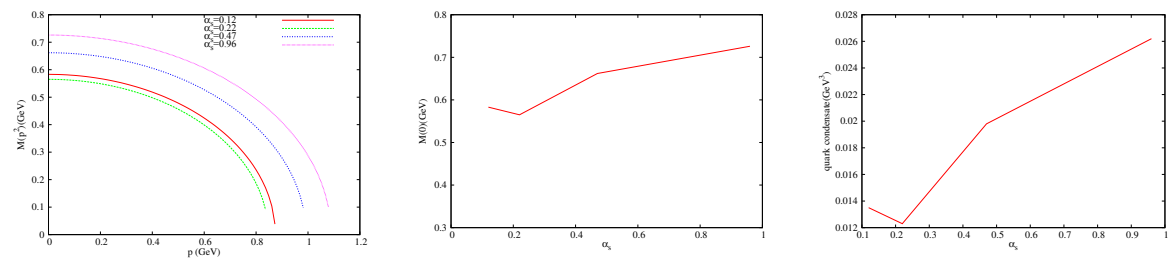

Figure 1. (Color online.) The variation $M\left(p^{2}\right)$ with $p, M(0)$ and $<q \bar{q}>$ with $\alpha_{s}$ for dual QCD vacuum.

and therefore a large amount of dynamical symmetry breaking seems to be brought on by the QCDmonopole condensation, which is responsible for the color confinement.

Further we study the manifestation of chiral symmetry at finite temperature in the dual QCD vacuum by making the following replacement in the solution of the SDE (10) of the dynamically generated quark-mass function. The solution at finite-temperature SDE in the limit $T \rightarrow 0$ is exactly reduced to the $\mathrm{SDE}$ at $T=0$. The quark mass function at finite temperature $\left(M_{T}\left(p^{2}\right)\right)$ is expressed as

$$
M_{T}\left(p^{2}\right)=\sqrt{\frac{2\left(m_{B}^{(T)}\right)^{2} \alpha_{s}}{\pi}-\frac{4 p^{2}}{9}},
$$

where $m_{B}^{(T)}$ is the thermal glueball masses which reduces monotonously near the critical temperature [20]. The variation of $M_{T}\left(p^{2}\right)$ at finite temperature is shown in Figure 2 for dual QCD vacuum and demonstrate a rapid decrease with temperature. The quark condensate at finite temperature $\left.(<q \bar{q}\rangle_{T}\right)$ using the $M_{T}\left(p^{2}\right)$ at finite temperature for dual QCD vacuum is obtained as

$$
<q \bar{q}>_{T}=-\frac{27}{40 \pi^{2}}\left(\frac{2\left(m_{B}^{(T)}\right)^{2} \alpha_{s}}{\pi}\right)^{3 / 2} .
$$

The ratio of $\left\langle\bar{q} q>_{T}\right.$ to that of $\left\langle\bar{q} q>_{T=0}\right.$ has been plotted as a function of temperature for dual QCD vacuum at $\alpha_{s}=0.47$ coupling in Figure 2 which gradually decreases with temperature and vanishes suddenly near the critical temperature, indicating the recovery of the chiral symmetry.
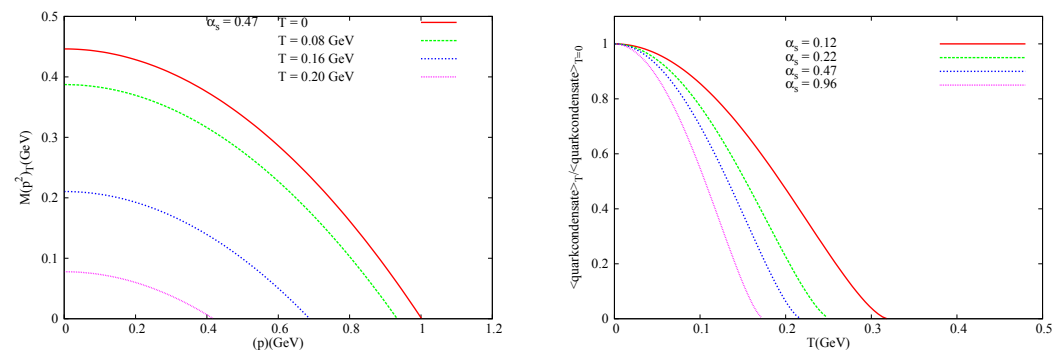

Figure 2. (Color online.) The variation of $M\left(p^{2}\right)$ with $p$ at different $T$ for $\alpha_{s}=0.47$ coupling and the ratio of $<\bar{q} q>_{T} /<\bar{q} q>_{T=0}$ as a function of $T$ for dual QCD vacuum at different coupling. 


\section{Results and Conclusions}

The mechanism of quark confinement has been studied in the context of a gauge theory of nonAbelian monopoles which describes an interesting dual dynamics between the color isocharge and the topological charge of a non-Abelian symmetry and allow an extra internal symmetry called magnetic symmetry which preserves the natural topological structure of the full gauge symmetry. In addition to the color confinement, the breaking of the chiral symmetry is also studied using Schwinger-Dyson techniques, characterized by large effective quark mass and quark condensate. The dependency of the quark mass function on the strong coupling constant and dual gluon mass has been established by solving the SDE for dual QCD and has been shown to lead to an intimate connection between confinement and dynamical chiral symmetry breaking in dual QCD vacuum. It has been shown that the strength of the dynamical chiral symmetry breaking increases in the deep infrared sector in dual QCD where the coupling are very intense. The recovery of the chiral symmetry breaking has also been discussed at finite temperature through the variation of the quark mass function and ratio of quark condensate at finite temperature to that at zero temperature which gradually decreases with temperature and vanishes suddenly near the critical temperature.

\section{Acknowledgments}

Garima Punetha, is thankful to the organizers of AYSS 2017 and University Grant Commission, New Delhi, India, under the UGC-RFSMS fellowship for financial assistance. Thanks are also due to referees for their valuable comments and suggestions.

\section{References}

[1] Y. Nambu, Phys. Rev. D10, (1974) 4262.

[2] G. 't Hooft, High Energy Physics edited by A. Zichichi (Editorice Compositori, Bologna, 1975).

[3] S. Mandelstam, Phys. Rep. 23, 245 (1976).

[4] C. N. Yang and R. L. Mills, Phys. Rev. 96, 191 (1954).

[5] R. Utiyama, Phys. Rev. 101, 1597 (1956).

[6] Y. M. Cho, Phys. Rev. D 21, 1080 (1980).

[7] Y. M. Cho, Phys. Rev. D 23, 2415 (1981).

[8] N. Cundy, Y. M. Cho, W. Lee, J. Leem, Phys. Lett. B 729, 192 (2014).

[9] H. C. Pandey and H. C. Chandola, Phys. Lett. B 476, 193 (2000).

[10] H. C. Chandola, Garima Punetha, H. Dehnen, Nucl. Phys. A 945, 226 (2016).

[11] Garima Punetha and H. C. Chandola, Springer Proceedings in Physics edited by Bhuyan Bipul, (Springer International Publishing, Switzerland, 2016) p. 653, Vol. 174.

[12] Garima Punetha and H. C. Chandola, EPL 116, 11001 (2016).

[13] H. J. Munczek and A. M. Nemirovsky, Phys. Rev. D 28, 181 (1983).

[14] D. W. Mckay and H. J. Munczek, Phys. Rev. D 55, 2455 (1997).

[15] P. C. Tandy, Prog. Part. Nucl. Phys. 39, 117 (1997).

[16] C. D. Roberts and A. G. Williams, Prog. Part. Nucl. Phys. 33, 477 (1994).

[17] A. C. Aguilar, A. A. Natale, and R. Rosenfeld, Phys. Rev. D 62, 094014 (2000).

[18] Alok Kumar, R. Parthasarathy, Phys. Lett. B 595, 373 (2004).

[19] I. A. Shushpanov, A.V. Smilga, Phys. Lett. B 402, 351 (1997).

[20] H. C. Chandola and D. Yadav, Nucl. Phys. A 829, 151 (2009). 